\title{
Priming during multiple-target search: The cumulative effects of relative target frequency
}

\author{
PATRICIA M. BLOUGH \\ Brown University, Providence, Rhode Island
}

\begin{abstract}
During search for multiple targets, reaction time decreases when a given target assumes a relatively high probability of appearance. Three experiments addressed the basis of this frequent-target effect. In a trialwise visual search method, pigeons pecked at the single target symbol embedded in a multisymbol display. In Experiment 1, the course of reaction times to frequent and infrequent targets was followed over extended sessions; reaction time to the frequent target dropped gradually over the first 200-400 trials. In Experiment 2, extinction sessions were introduced; the frequent-target effect persisted in the absence of reinforcement. In Experiment 3, the role of local trial patterns was examined; the frequent-target effect resisted temporary variations in relative target probability. Facilitation of search for high-probability targets appears to be an attentional rather than a conditioning effect; in the present design, information summarized over large sequences of trials appeared to provide pretrial information that primed attention.
\end{abstract}

During visual search, subjects attempt to detect certain items in a noisy visual field. Often there are several such targets. For example, a ground-feeding bird may search for a variety of acceptable food items among nonfood objects such as pebbles or dirt clumps. In the laboratory, subjects may learn to respond to several symbols constituting a target set and subsequently attempt to detect whichever of those symbols appears amid distractor symbols in a computer display.

In a multiple-target set, detection is facilitated when a particular target appears with a relatively high probability. For example, the foraging bird will prefer or will find more quickly an abundant food item (see, e.g., Bond, 1983; Tinbergen, 1960). When subjects search artificial displays, speed and accuracy are higher for more probable targets (P. M. Blough, 1992; Dykes \& Pascal, 1981). Accounts of this effect have emphasized selective attention, which comes to focus on the abundant item. The present experiments consider the searcher's ability to evaluate relative probabilities of events.

Attentional views stress the role of priming (Beller, 1971; P. M. Blough, 1989, 1991; Posner \& Snyder, 1975). Laboratory experiments in this tradition document improved search efficiency when there are advance cues to a target's identity. The work cited above shares the view that such cues activate an advance representation that facilitates detection. Naturalistic avian studies (e.g., Bond, 1983; Tinbergen, 1960) have also emphasized the role of

This research was supported by Grant 0407269 from the National Science Foundation. I thank Donna Lacourse for her assistance with the conduct of Experiment 1 and Donald Blough for many helpful suggestions. Address correspondence to the author, Department of Psychology, Box 1853, Brown University, Providence, RI 02912 (e-mail: patricia_blough@brown.edu). cues supplied by repeated encounters with a particular prey.

Although it is reasonable that high stimulus probability primes attention, it is not clear whether or how a subject translates relative frequency into pretrial information. A frequent target could be detected more efficiently because the stimulus is more likely to have appeared on the just preceding trial. For example, Bond (1983) proposed that, during foraging, an encounter briefly engaged a specific attentional mode that focused on the encountered prey. However, recent results (P. M. Blough \& Lacourse, 1994) failed to show an influence of the just-preceding trial. Alternatively, the subject could represent a short pattern of $n$ recent trials and translate this pattern into an informative cue. In a laboratory simulation of a rather different foraging problem, Shettleworth and Plowright (1992) found that pigeons' choices depended on very recent experience, described as a "memory window." Jarvik (1951) asked humans to predict forthcoming stimuli presented in a list. Despite the fact that the sequences were random, subjects' guesses depended on just-prior stimulus patterns.

It is also possible that subjects depend less on information provided by recent trials and more on relatively general rules. For example, the notion of optimal foraging behavior suggests that animals are guided by broad strategies that maximize benefits relative to costs (Kamil, 1984). In the laboratory, subjects learn rules pertaining to the overall experimental plan as well as specific trial rules (Honig, 1978; Olton, 1978). For example, in the studies of stimulus probability cited above, the identity of the frequent target typically varied between sessions. One appropriate strategy would lead the searcher to respond on the basis of accumulated experience over a lengthy series of early trials; the resulting representation might then resist local variations through the remainder of the session. 
Local patterns of search reaction times (RTs) should help distinguish among explanations of the frequenttarget effect. If a small subset of just-preceding trials provides the informative cue, RTs should depend on the particular targets presented in those trials. If pigeons learn more permanent rules, RTs to the frequent and infrequent targets should change gradually following a change in a condition, and RTs would then be relatively insensitive to local trial arrangements.

The present experiments were designed to assist in the evaluation of the accounts outlined above. In Experiment 1 , target probabilities were varied over many extended sessions. These data provided information about the course of changes in RT to frequent and infrequent targets following the introduction of differential probabilities. In Experiment 2, extinction tests were introduced in order to assess the role of reinforcement on the changes seen in Experiment 1. In Experiment 3, the persistence of the frequent-target effect was examined following the removal of differential probabilities. The combined data of the three experiments provided sufficient instances for analyses of local trial patterns and thus for the assessment of recent-trial effects.

\section{EXPERIMENT 1}

In Experiment 1, the detection of frequent and infrequent targets was assessed over the course of lengthy sessions. A replication of the frequent-target effect would show faster responses to the more probable target. Of special interest here was the manner in which this effect would emerge over trials.

\section{Method}

\section{Subjects}

The subjects were 3 male White Carneaux pigeons; all had been subjects in other search experiments with the same letter targets. The birds were maintained at $80 \%$ of their ad-lib feeding weights, and they typically collected their daily food ration during experimental sessions. They were housed in a pigeon colony room where they had free access to water with added vitamins and where a $17: 7-\mathrm{h}$ light:dark cycle was in effect.

\footnotetext{
Apparatus

Three experimental boxes were located in a separate room adjacent to that containing the controlling equipment. Fans ventilated the boxes, and a loudspeaker supplied masking noise. Each box enclosed a subject chamber $30 \mathrm{~cm}$ wide $\times 34 \mathrm{~cm}$ deep $\times 34 \mathrm{~cm}$ high . The chamber's front panel contained two horizontally centered openings. One aperture, centered $7 \mathrm{~cm}$ above the floor, was $5 \mathrm{~cm}$ wide $\times 4 \mathrm{~cm}$ high and provided access to a food tray when raised. A dim lamp was mounted above the food tray. The second opening, centered $18 \mathrm{~cm}$ above the floor, was $12 \mathrm{~cm}$ wide $\times 8.5 \mathrm{~cm}$ high and provided access to a $10.5-\mathrm{cm}$-wide $\times 6.7-\mathrm{cm}$-high monochromatic video screen. The screen was recessed by $3.2 \mathrm{~cm}$ and was surrounded by a Carroll touch frame. Programming software used the input from the touch frame to separate the screen into six equally wide vertical segments for the purpose of localizing keypecks. Controlling equipment included interfacing circuitry and IBM XT computers, which sensed responses, controlled experimental events, and collected data.
}

\section{Procedure}

The experiment was made up of 32 daily sessions, each consisting of 1,200 search trials. On each trial, a single target, drawn from a set of four, appeared among an array of distractors, and the pigeon's task was to peck at the target. Each session began with 12 uncounted warm-up trials and a subsequent 100 -trial baseline phase during which each target appeared equally often. During the remaining 1,100 test trials, a designated frequent target was displayed on $90 \%$ of the trials; the alternative targets appeared with equal probabilities on the remaining $10 \%$ of the trials. The computer's random statement determined these assignments. A session plan directed the identity of the frequent target. This plan organized the 32 sessions into eight blocks of 4 ; a different frequent target was designated on each of those 4 sessions, and this order changed between blocks. In addition, the session plan did not permit a given letter to be the frequent target on successive sessions.

Stimulus displays. Targets and distractors were black alphanumeric characters, $4 \mathrm{~mm}$ high and presented on a light background. A single display included 35 heterogeneous distractors and the selected target (the letters J, L, Q, or S). These items appeared as a $6 \times 6$ array with columns separated by $11 \mathrm{~mm}$ and rows by $9 \mathrm{~mm}$. The target's location was selected by a random-block method so that it appeared in each column equally often.

Response contingencies. Trials were separated by 1 -sec intertrial intervals (ITIs), during which the blank screen was set at its light background value. A response was defined as an uninterrupted sequence of two to four pecks to a given screen column. The length of this sequence differed among birds but was constant for each subject; its completion removed all display symbols. Following a response to the column containing the target, designated a correct detection, the screen dimmed briefly as a white bar appeared along the bottom. Usually the ITI then began. However, with a probability of .05 , a correct response also produced mixed-grain reinforcement for a duration that maintained the bird's running weight within the session; the white bar was present throughout this period. Following incorrect responses, the screen dimmed for a period of $7 \mathrm{sec}$, and there was no white bar. The trial was then rerun until a correct response occurred. Such rerun trials were not included in the data or trial counts.

Training. Although the pigeons had worked on similar search tasks (P. M. Blough \& Lacourse, 1994), some retraining was necessary. During these sessions, the four targets appeared with equal probability; display size was gradually increased and reinforcement probability decreased. Retraining ended when accuracy attained a minimum value of $90 \%$ at the final display size and reinforcement probability values. Final test sessions followed the session plan described above. However, if 3 or more days intervened between sessions, a review session was provided, in which all targets were presented with equal probability.

\section{Results}

Accuracy was high; birds typically performed at levels above $85 \%$ correct, and these scores were insensitive to experimental variables. The more informative measure was RT, indicating detection speed. The RTs reported below refer to the time between display onset and the first peck in the sequence that defined a correct response; analyses exclude RTs following errors. Summary values are medians. Overall, RTs to each of the four target letters differed, apparently reflecting differences in their discriminability. Experimental design and data analyses were planned so that each letter contributed equally to summary scores. Within-subject $t$ tests were used to evaluate the hypothesis that RT differences were equal to zero. 
The resulting $p$ values were evaluated according to a onetailed criterion, because, in each case, previous data and theory indicated a directional effect.

Table 1 describes the frequent-target effect, summarized over the 32 sessions comprised by the experiment. During baseline (left columns), the designated frequent target for the session occurred with the same probability as did the other three targets. RTs associated with frequent and infrequent targets were extremely close for all 3 birds. During the test phase (right columns ), the designated frequent target appeared on $90 \%$ of the trials. RTs associated with the frequent target were substantially lower for all 3 birds. This outcome, shown in more detail below, is consistent with the frequent-target effect described in the papers previously cited.

Further analysis addressed the course of RTs within sessions. For this purpose, a computer program evaluated within-session trials sequentially. The program scanned the data twice. The first scan limited its analysis to the frequent-target trials, grouping these into successive sets. An acceptable set was made up of eight trials and excluded errors and trials yielding RTs less than $0.1 \mathrm{sec}$. The second scan analyzed data for the infrequent targets. Here an acceptable set was made up of six trials, two associated with each infrequent target. Such sets also excluded errors and trials yielding RTs less than $0.1 \mathrm{sec}$; in addition, they excluded trials associated with a given target if that target was already represented twice in that set. Summary data combined such analyses over the 32 days and are expressed as within-set medians. Figure 1 shows RT changes over successive sets of test trials. Because the analysis excluded certain trial types, the actual trial on which an acceptable set was completed varied from session to session. For this reason, the values along the abscissa denote trial values for an ideal condition in which every trial provided an acceptable RT. Actual values would be higher.

The functions shown in Figure 1 reveal an early decline in RTs to the frequent target. RTs to the infrequent target also declined for 2 birds. The RT differential is consistent over trial sequences and substantiates the reliability of the frequent-target effect summarized in Table 1 . The gradual nature of the RT changes suggests effects of accumulating experience; if the frequent-target effect were attributable only to facilitating traces from

Table 1

Reaction Times to Frequent and Infrequent Targets (Experiment 1)

\begin{tabular}{lrrrrr}
\hline & \multicolumn{4}{c}{ Condition } \\
\cline { 2 - 4 } Bird & \multicolumn{2}{c}{ Baseline } & & Test \\
\cline { 2 - 6 } \cline { 2 - 5 } & Freq & Infreq & & Freq & Infreq \\
\hline B942 & 86 & 84 & 65 & 78 \\
B073 & 114 & 114 & 73 & 90 \\
B720 & 111 & 112 & 84 & 99 \\
$M$ & 104 & 103 & 74 & 89 \\
\hline
\end{tabular}

Note-Values are RTs in 1/100 sec. During baseline, the target labeled "frequent" had the same probability as did the others; this target was the more probable target during the subsequent test phase.
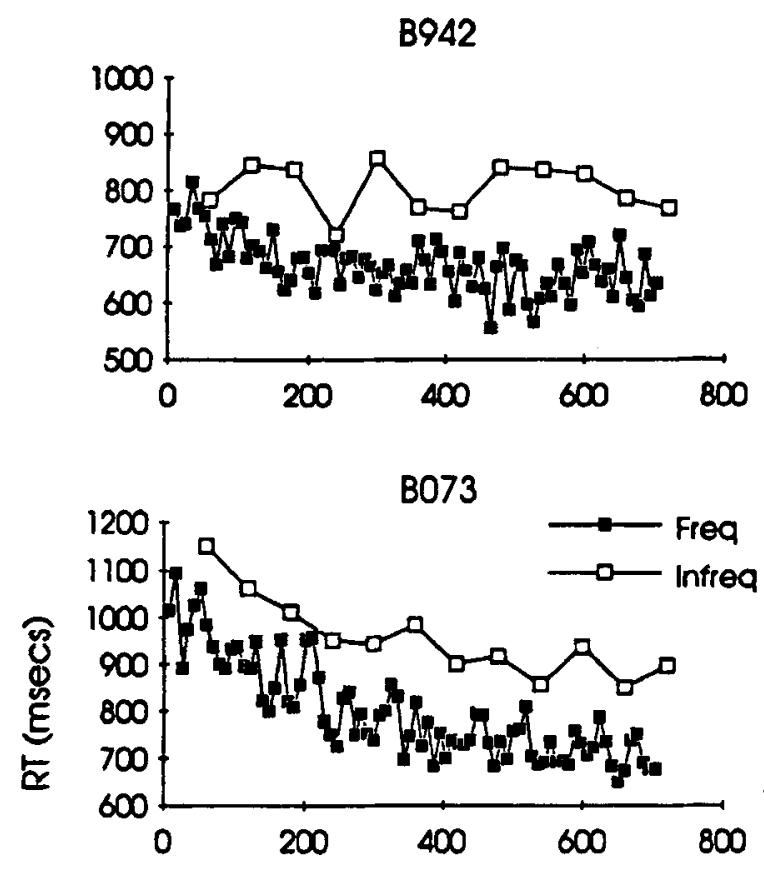

B720

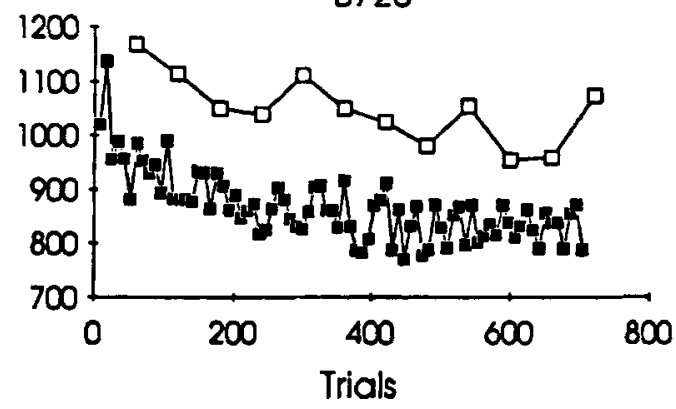

Figure 1. Follows the course of RTs to the frequent and infrequent targets. Data are combined over 32 sessions and exclude RTs less than $0.1 \mathrm{sec}$ and those associated with errors. For the frequent target, each data point summarizes a sequence of 8 trials; for the infrequent targets, each data point summarizes a sequence of 6 trials with the constraint that two RTs were associated with each of the three infrequent targets for that session. Trial values are appropriate to an ideal case; because of the constraints noted above and because the scheduling of trial types varied among sessions, these values are inexact. Each point shows a median over a minimum of 192 trials.

the previous trial, the reduction in associated RTs would have emerged more abruptly.

\section{EXPERIMENT 2}

The declining RT functions (Figure 1) suggest a learning curve. Indeed the data could be attributed to withinsession changes in associative strength--that is, to reinforcement occurring more often in the presence of the more probable target. In somewhat different paradigms, D. S. Blough $(1989,1992)$ demonstrated RT reductions associated with increased reinforcement probability. While we have noted problems with a reinforcement ac- 
count of the frequent-target effect (P. M. Blough, 1992; D. S. Blough, 1992), the evidence against it is indirect. Experiment 2 provided a more definitive approach to this issue through extinction tests.

\section{Method}

\section{Subjects}

The subjects were 5 birds. Three were the pigeons used in Experiment 1 ; the 2 additional birds had previously been subjects in search experiments with the same letter targets.

\section{Apparatus}

The apparatus was the same as that used in Experiment 1.

\section{Procedure}

The experiment was made up of 20 daily sessions. Of these, 16 were standard sessions that followed the program used in Experiment 1 . Following each 4-session block, there was a 700-trial extinction test. Such sessions, like the others, began with a 100-trial baseline phase during which all targets were equally probable and food reinforcement occurred with a probability of .05 . The remaining 600 trials were programmed as the previous test sessions were, but there was no food reward and no delay following errors. A different frequent target was assigned to each extinction test, and that target was never the frequent target assigned to the previous or following standard session.

As in Experiment 1, responses blanked the display and initiated the ITI. However, no visual feedback was associated with reward and errors in this experiment.

The stimulus displays, including the target and distractor symbols, were the same as those used in Experiment 1. The additional birds were retrained in the same manner as were the original 3 subjects. Occasionally there was an error in the conduct of the extinction test; in such cases, the four-session block was repeated before the test was redone.

\section{Results and Discussion}

Data analysis distinguished between baseline, control, and extinction conditions. Data from the baseline phases of all 16 nonextinction sessions contributed to the baseline analysis. The control analysis was based on the test phases of selected sessions, one from each block of four. The selected session was the one on which the frequent target was the same as that assigned to the extinction session following that block. Analysis of control sessions was restricted to the first 600 trials. Data from the test phases of all 4 extinction sessions contributed to the extinction analysis.

Table 2 summarizes the findings. During baseline, RTs to the target that would become the more probable were similar to those to targets that would be improbable in the subsequent trials. During both control and extinction conditions, there was a marked reduction in RTs associated with the frequent target compared with those associated with the infrequent targets. The size of the frequent-target effect is shown as the difference between RTs to the frequent and infrequent targets. Withinsubject $t$ tests tested the hypothesis that effect size was equal to zero. For baseline, $t(4)=1.20, p>.05$; for the control condition, $t(4)=5.86, p<.005$; for the extinction condition, $t(4)=2.76, p<.05$. This outcome establishes the significance of the frequent-target effect in extinction as well as during sessions with reinforcement.
Table 2

Reaction Times to Frequent and Infrequent Targets

(Experiment 2)

\begin{tabular}{|c|c|c|c|}
\hline Bird & Freq & Infreq & Diff \\
\hline \multicolumn{4}{|c|}{ Baseline } \\
\hline B942 & 99 & 98 & 1 \\
\hline B073 & 136 & 145 & -9 \\
\hline B720 & 87 & 91 & -4 \\
\hline B058 & 129 & 128 & 1 \\
\hline B374 & 83 & 82 & -1 \\
\hline$M$ & 107 & 109 & -2 \\
\hline \multicolumn{4}{|c|}{ Control } \\
\hline B942 & 75 & 92 & -17 \\
\hline B073 & 97 & 111 & -14 \\
\hline B720 & 81 & 94 & -13 \\
\hline B058 & 104 & 129 & -25 \\
\hline B374 & 76 & 81 & -5 \\
\hline$M$ & 87 & 101 & -14.8 \\
\hline \multicolumn{4}{|c|}{ Extinction } \\
\hline B942 & 79 & 90 & -11 \\
\hline B073 & 102 & 139 & -37 \\
\hline B720 & 90 & 103 & -13 \\
\hline B058 & 122 & 134 & -12 \\
\hline B374 & 86 & 99 & -13 \\
\hline$M$ & 96 & 113 & -17.2 \\
\hline
\end{tabular}

Note-Values are in 1/100 sec. Baseline: targets have equal probabilities. Control: frequent target appears with a probability of .9. Extinction: like control, but no reinforcement.

The pigeons responded differentially to frequent and infrequent targets in the absence of any primary reinforcement or differential visual feedback. Because the identity of the frequent target changed between sessions, the RT differences could not have carried over conditioning experienced on the previous day. In view of these findings, differential target-reinforcer associations cannot account for the frequent-target effect. The data are more consistent with attentional views that do not invoke motivationally significant events. However, such accounts note the costs as well as the benefits resulting from focused attention. The present data do not reveal evidence for such costs, which would be seen in elevated RTs to infrequent targets during control and extinction conditions compared with baseline (see Figure 1 and Tables 1-2). Perhaps, because baseline data came from the early trials of a long session, practice effects overshadowed such RT increases.

\section{EXPERIMENT 3}

Experiments 1 and 2 suggested that birds learn about relative target probabilities early in a session and revealed that such learning could occur in the absence of the usual reinforcing contingencies. Although it was evident that such learning took place gradually (Figure 1), the specific cues were not yet fully defined. In Experiment 3 , the persistence of this form of learning was examined following a withinsession change in target probabilities. This procedure also provided a more reliable measure of attentional costs. Furthermore, the data, when combined with those from Experiments 1 and 2, permitted evaluation of local trial patterns. 


\section{Method}

Subjects

The subjects were the participants in Experiment 2.

\section{Apparatus}

The apparatus was the same as that used in Experiments 1 and 2.

\section{Procedure}

There were 12 daily sessions. A session began with 12 warm-up trials with equal probabilities assigned to the four targets. A 600trial test phase followed immediately. As before, during each session a designated target appeared with a probability of .9 , with the other three targets assigned to the remaining trials with equal probability. The final phase was a 600 -trial baseline condition during which all targets appeared equally often. Food reinforcement occurred with a probability of .05 , and no differential visual feedback was associated with unreinforced correct and incorrect responses.

The session plan, stimulus displays, and other events were the same as those used in Experiment 1. Experiment 3 began as soon as the bird had completed Experiment 2.

B942

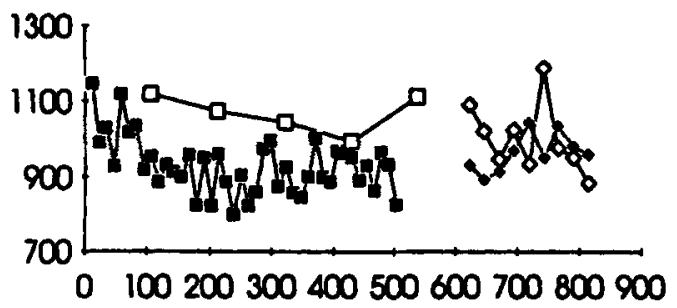

B720

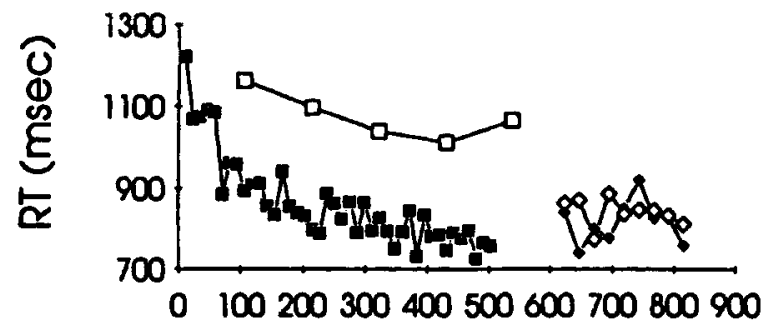

B374

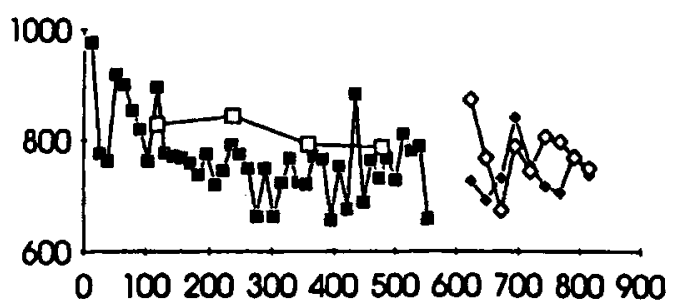

Trials

\section{Results and Discussion}

Figure 2 summarizes the data for both the test phase (left segments) and the subsequent baseline phase (right segments). The points referring to the test phase are based on 12-trial groups of acceptable frequent- and infrequenttarget trials. In the latter case, the group includes 4 trials with each infrequent target. The data, except for those from B058, conform well to the patterns seen in Experiment 1 . It is notable that the 2 less experienced birds (B058 and B374) showed more variability and a somewhat weaker frequent-target effect. A within-subject $t$ test established the significance of that effect. RTs to the frequent and infrequent targets were compared; $t(4)=3.64, p<.025$ by a directional test.

The right segments in each panel of Figure 2 show RTs to the previously frequent and infrequent targets following return to an equal-probability baseline. Individual points represent a sequence of 6 acceptable trials with the for-
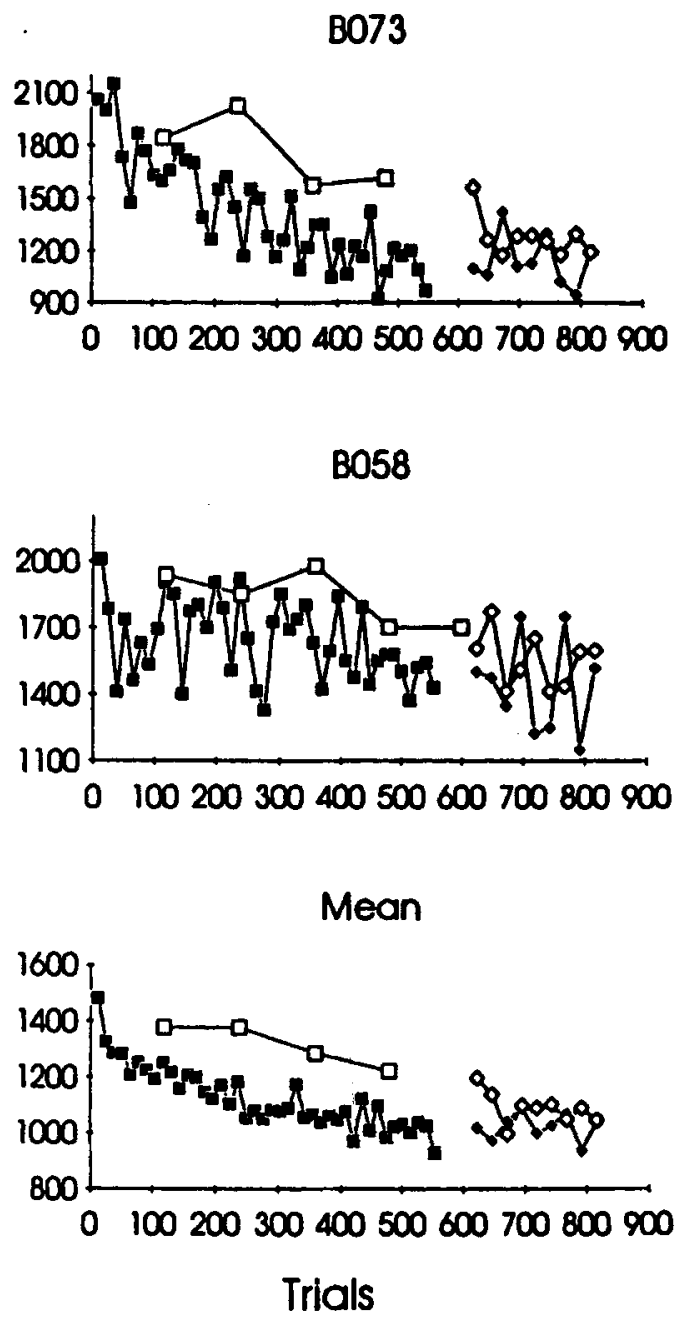

Figure 2. Follows the course of RTs to frequent (closed symbols) and infrequent targets (open symbols) during test and subsequent baseline phases. The baseline phase started on Trial 600 and is separated from the test phase by a gap. Data are combined over 16 sessions. For the test phase, each data point summarizes a sequence of 12 trials; for the infrequent targets, an acceptable sequence included four RTs from each of the three infrequent targets. For the baseline phase, a data point summarizes a 24-trial sequence with the constraint that it include six RTs associated with each target. Other constraints on analysis are the same as those described for Figure 1; trial numbering also followed the Figure 1 method. Each point shows a median over a minimum of 96 trials. 
merly frequent target and 18 acceptable trials ( 6 each) with the 3 formerly infrequent targets. To save space, the graph shows only a portion of this 600 -trial phase. The data, except for those from B720, show that the frequent-target effect persisted over the initial 48 or more trials of this phase, during which the formerly frequent target appeared with a probability of only .25 . Subsequent RTs, although variable, tended to converge for the two conditions.

These data indicate that the target-probability effect resists, for a while, marked changes in trial patterns. In the present design, sequences of two infrequent targets were unusual during the test phase. Sequences of three infrequent targets were extremely rare, although they were highly probable during baseline conditions. It appears that the pigeons relied less on information provided by a small set of just-preceding trials, but used a more extended series of events for cues to forthcoming target identity.

It is noteworthy that RTs to the infrequent targets tended to be higher during the test phase than they were during subsequent baseline. A within-subjects $t$ test evaluated this difference. Applied to the four data points common to all birds during the test phase and to 16 points during the baseline phase, $t(4)=2.59, p<.05$ by a directional test. This outcome is consistent with an attentional account, because it points to costs associated with attention directed elsewhere (Posner \& Snyder, 1975). In other words, enhanced attention to high-probability targets interfered with the detection of relatively improbable targets. Bond (1983) noted that such costs are difficult to explain in terms of associative models that emphasize reinforcement.

The data from the test phase, when pooled with data from Experiments 1 and 2, provided opportunities for evaluation of local effects. Figure 3 shows the combined data and the effects of just-preceding trials on the frequenttarget RT. These analyses were based on five-trial sequences terminating with the measured trial on which the frequent target appeared. A sequence was acceptable only if there were no errors and no reinforcement and if the RT fell between 0.1 and $4 \mathrm{sec}$. I hypothesized that such sequences provided pretrial information about the identity of the target on the measured trial, that less valid information would increase RT on that trial, and that such validity would depend on the number of infrequent targets appearing in the sequence and on the proximity of such trials to the measured trial. Figure 3 (top panel) refers to sequences when 0 or 1 infrequent target appeared and shows the position of the infrequent-target trial within the sequence. If an infrequent target reduced the validity of pretrial information, RT to the measured frequent target would be higher as the infrequent target grew closer to it in the sequence. Separate functions distinguish between effects over the entire session and effects over the first 400 trials, when RTs were declining. There appears to be no consistent pattern here; that is, RT to a frequent target did not appear to be affected by a recent infrequenttarget trial. The bottom panel of Figure 3 shows frequenttarget RTs as function of the number of infrequent targets in the preceding four-trial set. Again there appears to be no consistent pattern and thus no indication that an
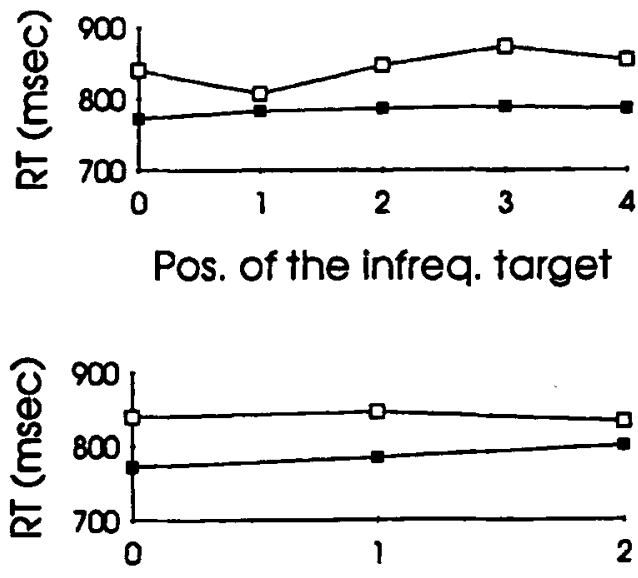

No. of infreq. targets

Figure 3. Shows means over 5 birds. Top panel: Median RT to a frequent target depending on the preceding 4-trial pattern. On the abscissa, Position 0 indicates no infrequent target in the pattern; Positions $1-4$ indicate the locus of a single infrequent-target trial, where 1 is the first and 4 is the most recent trial in the set. Bottom panel: Median RT to a frequent target depending on the number of infrequent targets during the preceding 4 trials. Data are pooled over the appropriate portions of Experiments 1-3. The figure separates functions that are based on entire sessions (closed symbols) from those based on the first 400 trials of each (open symbols). Each point shows a median over a minimum of 160 trials.

unusual density of infrequent-target trials impaired the validity of the facilitating cue. These analyses were limited in scope because there were insufficient instances of more extreme patterns. They are consistent, however, with the notion that the frequent-target effect survives short disruptions in same-target sequences.

\section{GENERAL DISCUSSION}

The present findings suggest certain bases for the frequent-target effect and cast doubt on others. Following introduction of a long trial series in which one target appeared with a very high probability, RT to the probable target declined gradually over 200-400 trials; overall, RT to more probable targets was substantially and reliably lower than it was to relatively improbable targets. The improved efficiency of detection was not attributable to differential strengthening of stimulus-reinforcer associations, because the frequent-target effect also occurred in extinction.

The findings are consistent with previous work that attributes the frequent-target effect to an attentional change (P. M. Blough, 1992; Bond, 1983; Reid \& Shettleworth, 1992). All three experiments show attentional benefits associated with increased predictability of a forthcoming event; Experiment 3 further revealed attentional costs associated with unexpected targets. The data also replicate and extend previous work, in which we failed to find carry-over effects from just-preceding trials (P. M. Blough \& Lacourse, 1994). We noted there that the bird's attention seems to be directed by a top-down process that uses 
advance information about target identity. It is interesting to contrast this conclusion with findings from human studies that show effects of very recent trials, even when they are not informative (Jarvik, 1951; Kirby, 1980). Bruner, Goodnow, and Austin (1956) suggested that such behavior may be attributable to superstitious guessing, possibly attributable to considerable experience with complex, but nonrandom, sequences of events. It is reasonable that pigeons should be less influenced in this way.

The present data suggest that attention was directed by an informative representation constructed over many trials. For example, as trials accumulate, summary codes, reflecting the occurrences of each target, may emerge. The informative value of such representations would depend on target incidence over a sizeable trial window. Thus, when a given target takes on a high probability, the associated representation becomes an increasingly valid predicter of the target to come, leading to gradually decreasing RTs. The code's basis on extended experience would account for its resistance to occasional contacts with infrequent target.

Similar accounts have been proposed in naturalistic foraging studies. Tinbergen (1960) used the term "searching image" to describe the representation associated with an abundant prey. In his words, "It may be supposed that such a specific searching image is assimilated when the birds have had a number of chance contacts with the species in question" (p. 316). In a study of preferences for cues associated with more and less abundant prey, Getty and Krebs (1985) noted that preferences initially did not reflect actual probabilities, but approached those probabilities over many trials. Their observations, identified as "lagging preferences" (p. 39), were attributed to sequential sampling, which moved a decision criterion in the direction of the more probable prey type. Getty and Krebs did find effects of the just-preceding trial events, but those events were more extended in time and involved the consumption of prey.

In Getty and Krebs's (1985) work, the preferences for cues signaling abundant prey could have been maintained by the food reward. In the present study, events other than reinforcement maintained the RT differential during extinction sessions. Ethological accounts implicate built-in adaptive strategies. For example, Guilford and Dawkins (1987) suggest ways in which attention directed toward abundant prey increases net gain. However, it is unclear whether this sort of explanation applies to the present work, which did not closely mimic natural search for food. A learning view might be more appropriate, given the pigeon's ability to learn general experimental rules (Honig, 1978; Olton, 1978). In the present studies, for example, one such rule involved the daily change in relative target probabilities. This rule was unchanged during extinction sessions. Experience with this program may have guided sampling processes that built an informative representation for that session.

\section{REFERENCES}

BeLler, H. K. (1971). Priming: Effects of advance information on matching. Journal of Experimental Psychology, 57, 176-182.

BLough, D. S. (1989). Contrast as seen in visual search reaction times. Journal of the Experimental Analysis of Behavior, 52, 199-211.

BLOUGH, D. S. (1992). Effects of stimulus frequency and reinforcement variables on reaction time. Journal of the Experimental Analysis of Behavior, 57, 47-50.

BLough, P. M. (1989). Attentional priming and visual search in pigeons. Journal of Experimental Psychology: Animal Behavior Processes, 15, 358-365.

Blough, P. M. (1991). Selective attention and search images in pigeons. Journal of Experimental Psychology: Animal Behavior Processes, 17, 292-298.

BLough, P. M. (1992). Detectability and choice during visual search: Joint effects of sequential priming and discriminability. Animal Learning \& Behavior, 20, 293-300.

Blough, P. M., \& Lacourse, D. M. (1994). Sequential priming in visual search: Contributions of stimulus-driven facilitation and learned expectancies. Animal Learning \& Behavior, 22, 275-281.

BoND, A. B. (1983). Visual search and selection of natural stimuli in the pigeon: The attention threshold hypothesis. Journal of Experimental Psychology: Animal Behavior Processes, 9, 292-306.

Bruner, J. S., Goodnow, J. J., \& Austin, G. A. (1956). A study of. thinking. New York: Wiley.

DyKes, J. R., JR., \& PASCaL, V. (1981). The effect of stimulus probability on the perceptual processing of letters. Journal of Experimental Psychology: Human Perception \& Performance, 7, 528-537.

GETTY, T., \& KREBS, J. R. (1985). Lagging partial preferences for cryptic prey: A signal detection analysis of great tit foraging. American Naturalist, 125, 29-60.

GUILFORD, T., \& DAWKINs, M. S. (1987). Search images not proven: A reappraisal of recent evidence. Animal Behaviour, 35, 1838-1845.

HONIG, W. K. (1978). Studies of working memory in the pigeon. In S. H. Hulse, H. Fowler, \& W. K. Honig (Eds.), Cognitive processes in animal behavior (pp. 211-248). Hillsdale, NJ: Erlbaum.

JARVIK, M. E. (1951). Probability learning and a negative recency effect in the serial anticipation of alternative symbols. Journal of Experimental Psychology, 41, 291-297.

KAMIL, A. C. (1984). Adaptation and cognition: Knowing what comes naturally. In H. L. Roitblat, T. G. Bever, \& H. S. Terrace (Eds.), Animal cognition (pp. 533-544). Hillsdale, NJ: Erlbaum.

KIRBY, N. (1980). Sequential effects in choice reaction times. In A. T. Welford (Ed.), Reaction times (pp. 129-172). New York: Academic Press.

Olton, D. (1978). Characteristics of spatial memory. In S. H. Hulse, H. Fowler, \& W. K. Honig (Eds.), Cognitive processes in animal behavior (pp. 341-373). Hillsdale, NJ: Erlbaum.

POSNER, M. I., \& SNYDER, C. R. R. (1975). Facilitation and inhibition in the processing of signals. In P. M. A. Rabbitt \& S. Dornic (Eds.), Attention and performance $V$ (pp, 669-682). New York: Academic Press.

ReID, P. J., \& Shettleworth, S. J. (1992). Detection of cryptic prey: Search image or search rate? Journal of Experimental Psychology: Animal Behavior Processes, 18, 273-286.

Shettleworth, S. J., \& Plowright, C. M. S. (1992). How pigeons estimate rates of prey encounter. Journal of Experimental Psychology: Animal Behavior Processes, 18, 219-235.

TINBERGEN, L. (1960). The natural control of insects in pinewoods: I. Factors influencing the intensity of predation by songbirds. Archives Neerlandaises de Zoologie, 13, 265-343.

(Manuscript received April 27, 1995; revision accepted for publication September 14, 1995.) 